\title{
Vacunación universal contra la varicela - Observación sobre ciertos cuestionamientos
}

\author{
Universal vaccination aganist chickenpox - Remarks on certain issues
}

Jerónimo Cello , Carlos García*

\begin{abstract}
Resumen
En este artículo, los autores revisan las evidencias actuales sobre ciertas observaciones epidemiológicas publicadas recientemente. En la publicación mencionada, el investigador argumenta que la vacunación universal contra la varicela no reduce el impacto de la enfermedad e incrementa el riesgo a largo plazo de glioma al evitar la infección natural con el virus de la varicela zóster. El análisis de las evidencias muestra que la vacunación universal reduce la morbilidad, la mortalidad y la tasa de hospitalizaciones relacionadas con la varicela. Más aún, y en contraste con lo sugerido, los datos demuestran que la vacunación universal induce un efecto rebaño.

Por otro lado, la información científica disponible sugiere que la asociación entre infección natural con virus de varicela zóster y un riesgo más bajo de padecer glioma es muy débil y no muestra causalidad. En este contexto debería considerarse que cualquier nivel de protección contra el glioma conferido por la infección natural es ampliamente superado por el impacto sobre la morbilidad y mortalidad causado por la varicela.

\section{Abstract}

In this article, the authors reviewed the current evidences for certain epidemiological observations published recently.

In the mentioned publication, the investigator claims that universal varicella vaccination does not reduce varicella burden and increases the long term risk of glioma by preventing natural infection with varicella zoster virus. The analysis of the evidences shows that universal vaccination reduces morbidity, mortality, and hospitalization related to varicella. In contrast to investigator's claim, moreover, the data demonstrate that universal vaccination does induce a herd effect.

On the other hand, the available scientific information suggests that the association between natural infection with varicella zoster virus and lower risk of glioma is very weak and does not show causality. In this context one should entertain the idea of any level of protection against glioma provided by natural infection with varicella zoster virus can be far outweighed by the impact on morbidity and mortality caused by chickenpox.
\end{abstract}

Cello J, Garcia C. Vacunación universal contra la varicela - Observación sobre ciertos cuestionamientos. Evid Act Pract Ambul. 2016;19(2):66-69. Abr-Jun.

Una publicación reciente de Internet titulada "Si ha pasado la varicela tiene menor probabilidad de padecer glioma" ha sido difundida en una lista de medicina familiar'.

La síntesis de dicha publicación concluye que la aplicación de la vacuna contra la varicela debería reservarse a niños de 12 años que no hayan padecido la enfermedad y que el padecimiento de varicela en la infancia es un cuadro leve que protege de por vida contra nuevos episodios evitando padecerla en edades más tardías o en el embarazo y, a su vez, reduciendo las posibilidades de padecer gliomas cerebrales'.

De esto se desprendería, como propone la publicación, que la vacunación contra la varicela debería excluirse del calendario obligatorio de vacunación y que esta exclusión, aplicando la vacuna en forma selectiva, disminuiría el riesgo de padecer gliomas cerebrales. Según el artículo, en España, en los años 2004 a 2006 se produjo solo una muerte en menores de 24 años mientras que ocurrieron 35 decesos en mayores de esa edad, remarcando que la varicela es especialmente grave en la mujer embarazada. El artículo remarca además que haber padecido la varicela deja una inmunidad permanente por lo que la enfermedad se padece una vez en la vida, que el virus persiste toda la vida en los ganglios sensitivos dorsales, pudiendo reactivarse en forma de herpes zóster (HZ), que luego de 40 años de experiencia de vacunación anti varicela en España los resultados no fueron convincentes respecto a su impacto en la morbilidad, que la vacuna se registró en España para su uso exclusivo en pacientes de alto riesgo en 1997, que en 2005 se recomendó su inclusión en el calendario de vacunación para niños de 12 años que no hubieran padecido la enfermedad, que el Ministerio de Salud cambió su criterio en la primavera de 2015 bajo la presión de algunos pediatras y de la Asociación Española de Pediatría y que si bien ciertas autoridades sanitarias se opusieron a este cambio de vacunación selectiva a vacunación universal, el negocio triunfo contra la ciencia prevaleciendo la conducta de vacunar mal (vacunación universal) sobre la vacunación selectiva. Más adelante, esta publicación manifiesta que el debate es importante dado que siendo una vacuna eficaz y necesaria el error en su administración (en forma universal) puede conllevar daños a la población y a la salud pública entre los que se contaría un incremento a largo plazo de la incidencia de gliomas cerebrales al haber, según múltiples estudios, una asociación inversa entre padecer la varicela y el riesgo a largo plazo de tal cáncer, por lo que padecer la enfermedad conferiría cierta protección contra el desarrollo de gliomas que sería disminuida por la aplicación de la vacuna. Menciona el estudio una publicación reciente de casos y controles de pacientes que padecieron gliomas en 14 centros de Dinamarca, Estados Unidos, Israel, el Reino Unido y Suecia en los que el precedente de haber padecido la varicela se asoció a una disminución del orden del $21 \%$ del riesgo de padecer gliomas y que esta asociación negativa es más fuerte para los gliomas malignos en individuos menores de 40 años. Además, según la misma publicación, estaría demostrado que el nivel de anticuerpos en sangre se asocia inversamente con el riesgo de padecer gliomas y que si bien no está claro el proceso biológico que pudiera originar esta protección, se propone que la infección natural por el virus de la varicela induciría al sistema inmune a eliminar células tumorales en etapas precoces. El autor del artículo comenta que la infección natural, produce inmunidad de por vida mientras que la inmunidad vacunal sería solo temporal y que en Estados Unidos se hizo evidente que era necesaria una segunda dosis de vacuna, que si bien no se sabe si esta doble vacunación produce una inmunidad de por vida sí se sabe que la varicela es más grave y tiene mayor mortalidad en adolescentes y adultos, y especialmente, en la mujer embarazada, por lo que considera que esta protección universal en la infancia podría tener consecuencias tardías graves e imprevisibles sugiriendo que sería mejor administrar la vacuna solo a los adolescentes que no han padecido la enfermedad (alrededor del $5 \%$ de ese grupo etario) evitando así introducir cambios en la historia natural de la misma que no son bien conocidos. Remarca además que la vacunación en la infancia disminuye los casos pero no las hospitalizaciones ni la mortalidad y que, contrariamente al efecto de otras vacunas, la vacuna contra la varicela produce un efecto contrario al efecto rebaño perjudicando a quienes no se vacunan que además, si la protección de los vacunados se desvanece con el tiempo, a largo plazo podrían dañarse también quienes se vacunan. Concluye esta publicación que la vacuna debería emplearse en los niños de 12 años que no padecieron la enfermedad y que

‡ Center for Infectious Diseases, Department of Molecular Genetics and Microbiology, School of Medicine, Stony Brook University jeronimo.cello@stonybrook.edu * Servicio de Medicina Familiar y Comunitaria del Hospital Italiano de Buenos Aires carlos.garcia@ hospitalitaliano.org.ar 
padecer la varicela en la infancia produce un cuadro leve que no solo protege de por vida contra la enfermedad, evitando su presentación en otras edades y situaciones, como el embarazo, sino que además disminuye el riesgo de padecer gliomas. Varias consideraciones y análisis en profundidad merece esta publicación.

\section{Impacto de la vacunación en la morbi-mortalidad por varicela} El mayor riesgo de complicaciones en adolescentes y adultos que padecen infecciones virales, comparado con el de los niños no es privativo de la varicela. La mayor agresividad de la enfermedad en etapas más tardías ocurre en varias y probablemente en todas las enfermedades virales como las causadas por el virus de Epstein Barr (VEB), el citomegalovirus (CMV), el virus de la rubéola (VR) y el virus de la parotiditis (VP) por mencionar solo algunos ejemplos. La mayor morbi-mortalidad de la varicela en jóvenes, adultos, reportada por Gérvas estaría dentro de lo esperable. Lo que no se informa en el comentario, que es fundamental para hacer una evaluación del impacto de la vacunación, es si la tasa de mortalidad en los diferentes grupos etarios aumentó o disminuyó respecto a otros períodos. En los Estados Unidos el incremento en la tasa de vacunación contra la varicela de niños de entre 19 y 35 meses de edad, de $27 \%$ en 1997 a $88 \%$ en 2005 produjo una reducción de entre $71 \%$ y $84 \%$ del número de casos, un $88 \%$ de las hospitalizaciones, un $59 \%$ de las visitas ambulatorias por cuidados relacionados con la varicela y un $92 \%$ de las muertes relacionadas con la misma $^{2,3}$. Estos datos además están avalados por cinco estudios de corte transversal realizados en Estados Unidos y en otros países que adoptaron la vacunación universal contra la varicela como Australia, Canadá, Alemania, Grecia, España y Uruguay $^{3,4}$. Por lo tanto estas evidencias no avalarían la sugerencia de que la vacunación no disminuye la morbi-mortalidad y hospitalizaciones causadas por la varicela ${ }^{1}$.

\section{Duración de la inmunidad conferida por la vacuna}

La información disponible en la actualidad indicaría que una dosis de vacuna generaría una inmunidad de larga duración, probablemente de por vida, en la mayoría de los vacunados y está documentado que entre un $90 \%$ y $97 \%$ de los individuos que han recibido una dosis de vacuna tienen anticuerpos contra el virus hasta al menos diez años después de haber sido vacunados $^{3,5}$. Las investigaciones realizadas sobre fallas de la vacuna en la inducción de inmunidad indican que la principal causa sería la incapacidad de la vacuna para inducir niveles protectores de inmunidad (falla primaria) y no una disminución de la inmunidad a lo largo del tiempo (falla secundaria) como sugiere Gérvas ${ }^{1,6}$

\section{Impacto epidemiológico y efecto rebaño de la vacunación contra la varicela}

La hipótesis de que al tener la varicela mayor morbi-mortalidad en adolescentes y adultos la inmunización universal de los niños podría retardar o de alguna forma desplazar la enfermedad a edades más tardías con consecuencias graves imprevisibles carece de sustento científico, dado que la vacunación contra la varicela en la infancia no solo no parece haber desviado la enfermedad a grupos etarios mayores de 11 años sino que además, con ella se ha observado una dramática disminución de la morbi-mortalidad en todos los grupos etarios ${ }^{7-10}$. En el caso de España, en Navarra, la aplicación desde 2006 de dos dosis disminuyó la incidencia de varicela en un $98 \% \%^{4,7}$.

Algunas publicaciones muestran que, en sociedades donde la cobertura es alta, se han observado dos patrones epidemiológi- cos de respuesta: a) un cambio en los grupos etarios con mayor incidencia de casos de varicela, de 3 a 6 años de edad a 9 a 11 años, y b) no cambio en el grupo etario con mayor incidencia (1 a 4 años de edad) $)^{6,8,11,9,12}$. Pero como se informa arriba, haya o no cambio en el grupo etario en que se observa la mayor incidencia de la enfermedad, la aplicación sistemática de la vacuna ha producido un disminución de los casos de varicela en todos los grupos etarios. Por lo cual el temor de que un cambio en el patrón epidemiológico debido a la vacunación causaría mayor morbi-mortalidad en adolescentes y adultos no parece tener correlato con las evidencias actuales. La razón de que no exista un aumento de casos de varicela en adolescentes y adultos radicaría en que, al estar dirigida la vacunación a la población con mayor incidencia de varicela, es decir a los niños menores de seis años, la circulación del virus disminuye notablemente en toda la población y en consecuencia las probabilidades de contagio disminuyen también en adolescentes y adultos ${ }^{3,13}$. Esta disminución de la circulación viral con consecuente menor afección de todos los grupos etarios por menor exposición es lo que se conoce como efecto rebaño inducido por la vacunación ${ }^{3,13}$. Una evidencia clara de que la inmunidad de rebaño (es decir, la inmunidad específica de los vacunados) inducida por la vacuna produce un efecto protector sobre el resto de la población no vacunada (efecto rebaño), son los estudios epidemiológicos de Baxter y colaboradores ${ }^{7}$. Estos investigadores demostraron que la aplicación sistemática de la vacuna contra la varicela disminuyó su incidencia no solo en la población vacunada sino también en la población no vacunada entre las edades 5 y 19 años desde un $18 \%$ en 1995 a un 5,8\% en 2009. Similar efecto rebaño protector inducido por la vacuna ha sido observado por otros investigadores ${ }^{4,10,12,14}$. Nuevamente, la información disponible contradice la hipótesis, sugerida por Gérvas de que la vacunación contra la varicela tendría un "efecto contrario a la protección de rebaño"1.

Inmunización contra varicela e incidencia de herpes zóster en la población adulta

La evidencia actual sugiere que los niños vacunados tienen una menor probabilidad de padecer herpes zoster $(\mathrm{HZ})$ que quienes se infectan por el virus salvaje, probablemente porque el virus salvaje tenga una mayor capacidad de reactivación que el virus atenuado vacunal ${ }^{15}$. Una de las especulaciones referentes a la administración de la vacuna a los niños sería que la vacuna disminuiría la circulación del virus en la población general la cual sería necesaria para inducir refuerzos en la inmunidad de la población adulta que tendrían como consecuencia el mantenimiento de niveles protectores de inmunidad que impedirían la reactivación del virus latente, evitando en consecuencia el HZ. Es decir, que la vacunación en niños, al disminuir la circulación viral aumentaría la incidencia de $\mathrm{HZ}$ en adultos. Existen estudios que muestran un aumento de la incidencia de $\mathrm{HZ}$ desde que se comenzó a aplicar la vacuna y otros estudios que evidenciarían lo contrario, es decir una disminución de la incidencia de $H Z^{3,16}$. Más aún, algunos estudios sugieren que el aumento de la incidencia del HZ comenzó antes de la era vacunal, principalmente porque la gente vive cada vez más y además hay más individuos sometidos a tratamientos inmunosupresores ${ }^{17}$. Analizando todos los resultados en conjunto habría habido un ligero aumento de la incidencia de $\mathrm{HZ}$ al comienzo de la era vacunal y datos de los últimos ocho años muestran que no ha habido incremento de la incidencia de $\mathrm{HZ}$ en el grupo de mayor riesgo constituido por individuos mayores de 65 años y se esperaría que con el aumento de la prevalencia de la población vacunada los casos disminuyan $^{3,16,18}$. Finalmente, el incremento aparente de la incidencia en la población adulta observado al principio fue muchísimo 
menor que el predicho por modelos matemáticos que consideraron como único refuerzo de la inmunidad contra el $\mathrm{HZ}$ a la excreción viral de los pacientes que padecen la varicela no teniendo en cuenta que los pacientes con $\mathrm{HZ}$ también eliminan el virus y que existen además reactivaciones endógenas asintomáticas que inducen una reactivación de la inmunidad específica que contribuye a disminuir la incidencia de $\mathrm{HZ}$ en la población en riesgo ${ }^{3}$.

\section{Vacunación universal versus vacunación selectiva a gru- pos de alto riesgo}

La estrategia de vacunación selectiva contra la varicela, a grupos de alto riesgo, es decir adolescentes que no han padecido la enfermedad, fundamentalmente para evitar la enfermedad en grupos en los que la morbi-mortalidad es mayor, y en las mujeres embarazadas para prevenir la varicela congénita $y$ perinatal ha fallado en muchas oportunidades, entre otras cosas por la dificultad que implica acceder a estos grupos de mayor riesgo puesto que, como ha sido bien documentado los adolescentes constituyen un grupo donde los cuidados preventivos son muy deficientes ${ }^{19-21}$. En las sociedades con un alto índice de vacunación, como es el caso de España, correspondería en primer lugar considerar si las bajas tasas de mortalidad no son un resultado positivo de la inmunización, en lugar de considerar que en caso de una vacunación selectiva disminuiría aún más la mortalidad más allá de los 24 años.

\section{Costo efectividad de la vacunación contra la varicela}

Existen varios estudios que demuestran que los programas de vacunación contra la varicela son costo-efectivos ${ }^{13}$. De todos modos estos estudios se realizaron con los programas de una dosis por lo que si la duplicación de la dosis no se compensara por una reducción de la falla vacunal primaria el impacto en la costoefectividad sería negativo. El régimen de vacunación con dos dosis se mostró costo-efectivo cuando se lo comparó con la no vacunación pero no cuando se lo comparó con el de una dosis ${ }^{22}$. Es importante comprender que hay una considerable variabilidad de la metodología utilizada en los diferentes estudios que impacta sobre los resultados. El tipo de modelo utilizado (estático versus dinámico), la perspectiva examinada (desde los proveedores o desde la sociedad), la inclusión del efecto rebaño y cobertura vacunal y los diferentes precios y salarios parentales tienen un significativo impacto en los resultados computarizados ${ }^{23}$.

\section{Varicela y glioma}

Según diferentes investigaciones existiría una asociación inversa entre el padecimiento de varicela y el riesgo de padecer gliomas ${ }^{24-28}$. Basándose en estas observaciones, se postula que la vacunación universal cambiaría la historia natural de la enfermedad y no infectarse con el virus salvaje implicaría un daño potencial pues conllevaría un incremento a largo plazo de la incidencia de dicho tumor. En aras de protegerse contra una mayor incidencia de glioma se sugiere entonces la vacunación selectiva. La especulación científica subyacente a la protección conferida por la varicela contra el desarrollo de glioma sería que la respuesta inmune montada contra el virus de la varicela zóster (VVZ) induciría una reacción inmunológica cruzada que reconocería epítopes en las células tumorales y las eliminaría. Una explicación alternativa sugiere que el virus de la varicela tiene capacidad de regular el desarrollo de glioma mediante un mecanismo de infección crónica e inflamación ${ }^{25}$. El grado de evidencia de que los pacientes que sufrieron varicela tienen menor riesgo de gliomas es muy bajo. Un estudio concluye que los pacientes que han padecido la varicela tienen un riesgo relativo $21 \%$ menor de padecer gliomas cerebrales y que este efecto protector de la enfermedad fue más fuerte para los gliomas de alto grado ${ }^{24}$. El diagnostico de varicela en este estudio fue realizado retrospectivamente por medio de cuestionarios. Se debe considerar que el diagnóstico retrospectivo de infección natural por varicela por medio de cuestionarios no es confiable y en pacientes portadores de tumores cerebrales que tienen concurrente deterioro cognitivo puede ser aún menos confiable $^{29}$. Es importante remarcar que, siendo la incidencia poblacional de gliomas del orden de 5 por 100.000 adultos $(0,005 \%)$ una reducción relativa de $21 \%$ del riesgo implica bajar esa incidencia en 1 por cada 100.000 individuos es decir a 4 por 100.000 adultos o 0,004\% a expensas, probablemente, de tener más casos, más internaciones y más muertes por varicela ${ }^{25}$. Constituyen otra línea de evidencia los estudios que muestran que los pacientes que tienen menores niveles de anticuerpos contra el VVZ tienen mayor riesgo de padecer glioma ${ }^{25-28}$. Sin embargo, la detección de anticuerpos contra el virus de la varicela, tanto en los pacientes con glioma como en aquellos que no lo padecieron, indican que ambos grupos se infectaron con el virus, por lo que una explicación más plausible a la relación inversa entre varicela y desarrollo de gliomas sugerida por los investigadores sería que los pacientes portadores de gliomas podrían tener un sistema inmune deficiente, incapaz de controlar en forma eficiente el desarrollo tumoral y que los menores niveles de anticuerpos detectados contra el virus de varicela serian simplemente un indicador de esa inmunodeficiencia ${ }^{24}$. En algunos de los trabajos mencionados previamente, además de los anticuerpos contra VVZ se midieron los niveles de anticuerpos contra CMV, VEB, virus del herpes simple (VHS), y adenovirus (ADV). Los investigadores observaron que la relación inversa entre niveles de anticuerpos específicos contra esos virus y riesgo de padecer glioma era menos frecuente que la relación inversa con VVZ, lo que sugeriría algún grado de asociación entre infección por el VVZ y el menor riesgo de tener glioma ${ }^{26-28}$. Por otra parte, Poltermann y colaboradores estudiaron la sero-prevalencia de anticuerpos contra CMV, VHS, VEB y VVZ y no encontraron diferencias significativas entre pacientes con gliomas y población general ${ }^{30}$. Un dato a tener muy en cuenta es que los niveles de anticuerpos en ciertos trabajos fueron determinados en no pocos pacientes que habían recibido tratamientos inmunosupresores que podrían inducir una menor producción de anticuerpos ${ }^{26-28}$. De hecho, cuando los pacientes previamente tratados con esteroides $u$ otros inmunosupresores no fueron incluidos, la asociación inversa fue menos marcada ${ }^{27}$. El HZ o simplemente zóster es producido por la reactivación del VVZ, que permanece en estado latente después de la infección primaria en los ganglios nerviosos. Por lo tanto los pacientes con $\mathrm{HZ}$ han padecido varicela en algún momento de sus vidas. Algunos estudios muestran un riesgo incrementado de tumores malignos o una peor evolución de tumores hematológicos en los pacientes que padecen $\mathrm{HZ}^{31-33}$. Basándose en estas observaciones se puede especular que existiría una asociación positiva entre varicela y un mayor riesgo de padecer diferentes tipos de cánceres. Sin embargo, otros estudios, no fueron capaces de demostrar esta asociación ${ }^{34,35}$.

\section{Conclusiones}

La vacunación universal contra la varicela ha sido muy efectiva y ha disminuido dramáticamente la morbi-mortalidad en todos los grupos etarios así como también las hospitalizaciones asociadas a la enfermedad. La hipótesis de un desplazamiento de la enfermedad a grupos etarios más vulnerables y el consiguiente aumento de la morbi-mortalidad en adolescentes y 
adultos no ha sido demostrada en absoluto. Tampoco se observó un aumento apreciable en la incidencia de casos de $\mathrm{HZ}$ en la población adulta como consecuencia de la vacunación en los niños. Además, la vacuna parece producir un efecto rebaño que protege a la población no inmunizada, evidencia que contradice la especulación de que la vacunación contra la varicela induciría un "efecto rebaño negativo". No existen dudas de que la vacunación con una o dos dosis es costo-efectiva cuando se la compara con no vacunar. Sin embargo, la costo-efectividad de las diferentes modalidades de vacunación aún no ha sido completamente estudiada y para su análisis deben incluirse muchos factores que actúan en los múltiples y variados escenarios sociales analizados. Con respecto a los gliomas, hasta el momento los únicos factores confirmados que incrementan el riesgo padecerlos son las radiaciones ionizantes y los factores hereditarios predisponentes, siendo las demás teorías débiles y meramente especulativas ${ }^{30}$. La existencia de una asociación inversa entre varicela y el riesgo de padecer gliomas está basada en un nivel de evidencia científica insuficiente, incapaz de sustentar una relación causal entre infección por VVZ y riesgo de gliomas. La explicación de los mecanismos inmunológicos inducidos por la infección con VVZ que conferirían protección contra el desarrollo de gliomas es altamente especulativa, más aún considerando que los estudios serológicos indican que la mayoría de los pacientes con gliomas también habían padecido varicela. Dado el bajo grado de evidencia de la relación inversa entre varicela y riesgo de padecer glioma, la hipótesis de la vacunación selectiva como elemento de protección contra el padecimiento futuro de gliomas es muy poco sustentable. Igualmente, la hipótesis de que la vacunación universal contra la varicela causaría un aumento de la incidencia a largo plazo de gliomas cerebrales es inconsistente y de ocurrir, el aumento absoluto de la incidencia sería marginal. Además, debería considerarse que si la vacunación selectiva es menos efectiva que la universal contra la infección por VVZ, el incremento de la morbi-mortalidad por varicela podría superar este supuesto beneficio de una disminución de los casos de glioma. Una pregunta científica válida de ser formulada es que si la infección por virus salvaje disminuye el riesgo de padecer glioma, por qué la infección vacunal, que simula a la natural, no habría de disminuirlo.

Recibido el 06/08/2016 y aceptado el 10/08/2016

Referencia:

1. Gévas J. Si ha pasado la varicela tiene menor probabilidad de padecer glioma (cáncer de cerebro) [Intemet]. 2016. Disponible en: http://www.actasanitaria.com/si-ha-pasado-la-varicela-tiene-menorprobabilidad-de-padecer-glioma-cancer-de-cerebro/ (útimo acceso julio 2016)

2. American Academy of Pediatrics Committee on Infectious Diseases. Prevention of varicella: recommendations for use of varicella vaccines in children, including a recommendation for a routine 2 dose varicella immunization schedule. Pediatrics. $2007 \mathrm{Jul} ; 120(1): 221-31$. Disponible en: http://www.ncbi.nlm.nih.gov//pubmed/17606582

3. Papaloukas O, y col. Successes and challenges in varicella vaccine. Ther Adv vaccines [Intemet]. 2014 Mar;2(2):39-55. Disponible en: http://www.ncbi.nlm.nih.gov/pubmed/24757524

4. García Cenoz M, y col. Impact of universal two-dose vaccination on varicella epidemiology in Navarre, Spain, 2006 to 2012. Euro Surveill Bull Eur sur les Mal Transm = Eur Commun Dis Bull [Intemet]. 2013;18(32):20552. Disponible en: http://www.ncbi.nlm.nih.gov/pubmed/23968827

5. Communication and Education Branch NC for I, and Respiratory Diseases C for DC and P. EPIDEMIOLOGY AND PREVENTION OF VACCINEPREVENTABLE 13 DISEA TH EDITION SES [Intemetl. 2015. Disponible en: https://www.cdc.gov/vaccines/pubs/pinkbook/downloads/table-of-contents.pdf

6. Bonanni P, y col. Primary versus secondary failure after varicella vaccination: implications for interval between 2 doses. Pediatr Infect Dis J [intemet]. 2013 Jul;32(7):e305-13. Disponible en: http://www.ncbi.nlm.nih.gov/pubmed/23838789

7. Baxter R, y col. Impact of vaccination on the epidemiology of varicella: 1995-2009. Pediatrics [Internet]. 2014 Jul;134(1):24-30. Disponible en: http://mww.ncbi.nlm.niih.gov/pubmed/24913796 8. Seward JF, y col. Varicella disease after introduction of varicella vaccine in the United States, 1995-2000. JAMA [Internet]. 2002 Feb 6;287(5):606-11. Disponible en: http://www.ncbi.nlm.nih.gov/pubmed/11829699

9. Chaves SS, y col. Loss of vaccine-induced immunity to varicella over time. N Engl J Med [Internet]. 2007 Mar 15;356(11):1121-9. Disponible en: http://www.ncbi.nlm.nih.gov/pubmed/17360990

10. Siedler A, Amdt U. Impact of the routine varicella vaccination programme on varicella epidemiology in Germany. Euro Surveill Bull Eur sur les Mal Transm $=$ Eur Commun Dis Bull [Internet] 2010;15(13). Disponible en: http://www.ncbi.nlm.nih.gov/pubmed/20394710

11. García Cenoz M. Varicella vaccination programs do not seem to shift the age of disease to older age groups. Transl Pediatr [Internet]. 2014 Oct;3(4):275-7. Disponible en: http://www.ncbi.nlm.nih.gov/pubmed/26835346

12. Quian J, y col. Impact of universal varicella vaccination on 1-year-olds in Uruguay: 1997-2005. Arch Dis Child [Internet]. 2008 Oct;93(10):845-50. Disponible en: http://www.ncbi.nIm.nih.gov/pubmed/18456699

13. Bonanni P, y col. Varicella vaccination in Europe - taking the practical approach. BMC Med [Internet]. 2009;7:26. Disponible en: http://www.ncbi.nlm.nih.gov/pubmed/19476611

14. Giammanco G, y col. Universal varicella vaccination in the Sicilian paediatric population: rapid uptake of the vaccination programme and morbidity trends over five years. Euro Surveill Bull Eur sur les Mal Transm = Eur Commun Dis Bull [lnternet]. 2009;14(35). Disponible en: http://www.ncbi.nlm.nih.gov/pubmed/19728978

15. Schmid DS, Jumaan AO. Impact of varicella vaccine on varicella-zoster virus dynamics. Clin Microbiol Rev [Internet]. 2010 Jan;23(1):202-17. Disponible en: http://www.ncbi.nlm.nih.gov/pubmed/20065330

16. Baxter R. Impact of vaccination on the epidemiology of varicella. Transl Pediatr [Internet]. 2015 Jan;4(1):63-4. Disponible en: http://www.ncbi.nlm.nih.gov/pubmed/26835362

17. Joesoef RM1, y col. Chronic medical conditions as risk factors for herpes zoster. Mayo Clin Proc. 2012. p. 961-7. Disponible en: http://www.ncbi.nlm.nih.gov/pubmed/?term=Joesoef+Rm+961

18. Weinmann S, y col. Incidence and clinical characteristics of herpes zoster among children in the varicella vaccine era, 2005-2009. J Infect Dis [Internet]. 2013 Dec 1;208(11):1859-68. Disponible en: http://www.ncbi.nlm.nih.gov/pubmed/23922376

19. Sengupta N, y col. Varicella vaccination in Europe: are we ready for a universal childhood programme? Eur J Pediatr [Internet]. 2008 Jan;167(1):47-55. Disponible en: http://www.ncbi.nlm.nih.gov/pubmed/17334784

20. Mongua-Rodriguez N1, y col. A systematic review of rubella vaccination strategies implemented in the Americas: impact on the incidence and seroprevalence rates of rubella and congenital rubella syndrome. Vaccine [Internet]. 2013;31(17):2145-51. Disponible en: http://www.ncbi.nlm.nih.gov/pubmed/?term=Vaccine.+2013+Apr+19\%3B31(17)\%3A2145-51.

21. Van Damme P, y col. Integration of hepatitis B vaccination into national immunisation programmes. Viral Hepatitis Prevention Board. BMJ [Intemet]. 1997 Apr 5;314(7086):1033-6. Disponible en: http://www.ncbi.nlm.nih.gov/pubmed/9112852

22. Zhou F, y col. Impact of varicella vaccination on health care utilization. JAMA [Intemet]. 2005 Aug 17;294(7):797-802. Disponible en: http://www.ncbi.nIm.nih.gov/pubmed/16106004 23. Soárez PC de, y col. Impact of methodology on the results of economic evaluations of varicella vaccination programs: is it important for decision-making? Cad saúde pública [Intemet]. 2009;25 Suppl 3:S401-14. Disponible en: http://www.ncbi.nlm.nih.gov/pubmed/20027388

24. Amirian ES, y col. History of chickenpox in glioma risk: a report from the glioma international case-control study (GICC). Cancer Med [Intemet]. 2016 Jun;5(6):1352-8. Disponible en: http://www.ncbi.nlm.nih.gov/pubmed/26972449

25. Lee S-T, y col. Interaction of allergy history and antibodies to specific varicella-zoster virus proteins on glioma risk. Int J cancer [Internet]. 2014 May 1;134(9):2199-210. Disponible en: http://www.ncbi.nlm.nih.gov/pubmed/24127236

26. Wrensch M, y col. Prevalence of antibodies to four herpesviruses among adults with glioma and controls. Am J Epidemiol [Internet]. 2001 Jul 15;154(2):161-5. Disponible en: http://www.ncbi.nlm.nih.gov/pubmed/11447050

27. Wrensch M, y col. History of chickenpox and shingles and prevalence of antibodies to varicella-zoster virus and three other herpesviruses among adults with glioma and controls. Am J Epidemiol [Intemet]. 2005 May 15:161(10):929-38. Disponible en: http://www.ncbi.nlm.nih.gov/pubmed/15870157

28. Sjöström S, y col. Human immunoglobulin G levels of viruses and associated glioma risk. Cancer Causes Control [Internet]. 2011 Sep;22(9):1259-66. Disponible en: 28. Sjöström S, y col. Human immunoglob

29. Taphoorn MJ1 KM. Cognitive deficits in adult patients with brain tumours. Lancet Neurol [Internet]. 2004;3(3):159-68. Disponible en: http:/www.ncbi.nlm.nih.gov/pubmed/14980531

30. Poltermann S, y col. Lack of association of herpesviruses with brain tumors. J Neurovirol [Internet]. 2006 Apr;12(2):90-9. Disponible en: http://www.ncbi.nlm.nih.gov/pubmed/16798670

31. Sørensen HT, y col. The risk and prognosis of cancer after hospitalisation for herpes zoster: a population-based follow-up study. Br J Cancer [Internet]. 2004 Oct 4;91(7):1275-9. Disponible en: http://www.ncbi.nlm.nih.gov/pubmed/15328522

32. Ho JD1, Xirasagar S LH. Increased risk of a cancer diagnosis after herpes zoster ophthalmicus: a nationwide population-based study. Ophthalmology [Internet]. 2011;118(6):1076-81. Disponible en: http://mww.ncbi.nlm.nih.gov/pubmed/?term=Ophthalmology+2011\%3B+118(6)\%3A+1076-81.

33. Cotton SJ, y col. The risk of a subsequent cancer diagnosis after herpes zoster infection: primary care database study. Br J Cancer [Internet]. 2013 Feb 19;108(3):721-6. Disponible en: http://www.ncbi.nlm.nih.gov/pubmed/23361054

34. Buntinx F, y col. Is herpes zoster a marker for occult or subsequent malignancy? Br J Gen Pract [Internet]. 2005 Feb;55(511):102-7. Disponible en: http://www.ncbi.nIm.nih.gov/pubmed/15720930 35. Wang Y-P, y col. Risk of cancer among patients with herpes zoster infection: a population-based study. CMAJ [Internet]. 2012 Oct 16;184(15):E804-9. Disponible en: http://www.ncbi.nlm.nih.gov/pubmed/22988158 